\title{
Association between monoallelic TSHR mutations and congenital hypothyroidism: a statistical approach
}

\author{
Kiyomi Abe', Satoshi Narumi 1,2, Ayuko S. Suwanai', Masanori Adachi' ${ }^{3}$, Koji Muroya ${ }^{3}$, Yumi Asakura ${ }^{3}$, \\ Keisuke Nagasaki ${ }^{4}$, Takayuki Abe ${ }^{5}$ and Tomonobu Hasegawa1
}

${ }^{1}$ Department of Pediatrics, Keio University School of Medicine, Tokyo, Japan, ${ }^{2}$ Department of Molecular Endocrinology, National Research Institute for Child Health and Development, Tokyo, Japan, ${ }^{3}$ Department of Endocrinology and Metabolism, Kanagawa Children's Medical Center, Yokohama, Japan, ${ }^{4}$ Division of Pediatrics, Department of Homeostatic Regulation and Development, Niigata University Graduate School of Medicine and Dental Sciences, Niigata, Japan, and ${ }^{5}$ Center for Clinical Research, Keio University School of Medicine, Tokyo, Japan

Correspondence should be addressed to S Narumi

Email

narumi-s@ncchd.go.jp

\begin{abstract}
Objective: Biallelic TSHR mutations cause congenital hypothyroidism (CH). Serum TSH levels of monoallelic mutation carriers range from normal to mildly elevated, and thus the size of its effect remains unclear. The objectives were to examine the association between monoallelic TSHR mutations and positivity at newborn screening (NBS) for CH, and to test whether the association was modified by another genetic factor.

Subjects and methods: We enrolled 395 patients that had a positive result in NBS and sequenced TSHR. Monoallelic TSHR mutation carriers were further sequenced for DUOX2. Molecular functions of the mutations were verified in vitro. The frequency of the mutations in the study subjects was compared with a theoretical value in the Japanese general population. Odds ratio (OR) for NBS positivity associated with the mutation was calculated. Using Bayes' theorem, we estimated a posterior probability of NBS positivity given the mutation.

Results: Twenty-six monoallelic TSHR mutation carriers were found. Four out of the 26 also had a monoallelic DUOX2 mutation (double heterozygotes). The frequencies of monoallelic TSHR mutation carriers (6.6\%) and double heterozygotes (1.0\%) were significantly higher than those in the general population $(0.58 \%$ and $0.0087 \%$, respectively). OR for NBS positivity of having a monoallelic TSHR mutation or being a double heterozygote was 12.0 or 117.9 , respectively. Posterior probability of NBS positivity was $0.38 \%$ in monoallelic TSHR mutation carriers and $3.8 \%$ in double heterozygotes.

Conclusions: Monoallelic TSHR mutations are significantly associated with NBS positivity, and the association is further strengthened by the coexistence of monoallelic DUOX2 mutations.
\end{abstract}

\section{Introduction}

Congenital hypothyroidism $(\mathrm{CH})$ is the deficiency of thyroid hormone production that affects infants from birth. Thyroid hormone deficiency in early infancy leads to irreversible intellectual disability. Because early diagnosis and treatment can prevent the disability, most developed countries have introduced newborn screening (NBS) for $\mathrm{CH}$ (1). The implementation of $\mathrm{CH}$ screening has enabled physicians to find mild $\mathrm{CH}$ cases (also known as hyperthyrotropinemia) in which no obvious $\mathrm{CH}$-related pathologies are observed (2). However, the pathogenesis underlying such mild $\mathrm{CH}$ cases remains largely unknown, and we hypothesize that multiple genetic factors are involved. www.eje-online.org

https://doi.org/10.1530/EJE-16-1049
(C) 2018 European Society of Endocrinology Printed in Great Britain
Published by Bioscientifica Ltd. 
The thyroid hormone-producing capacity is regulated by thyroid-stimulating hormone (TSH)/ TSH receptor signaling. Sunthornthepvarakui and coworkers (3) reported that $\mathrm{CH}$ occurred by inactivating TSH receptor gene (TSHR) mutations that follows autosomal recessive inheritance. Currently, more than $50 \mathrm{CH}$ patients with biallelic TSHR mutations have been reported (4). Additionally, more than 100 cases with monoallelic (i.e. heterozygous) TSHR mutation, including parents and siblings of patients with biallelic TSHR mutation, have been reported $(5,6,7,8,9,10)$. Approximately $20 \%$ of the reported monoallelic TSHR mutation carriers had moderate or high-serum TSH levels $(10-70 \mathrm{mU} / \mathrm{L})$ (7, $8,9,10)$. Another evidence linking monoallelic TSHR mutations and TSH elevation is frequent detection of monoallelic mutation carriers in patients with nonautoimmune hypothyroidism $(8,9,11)$. In several reports, TSHR mutations were regarded as an autosomal dominant trait $(12,13)$.

Considering that the frequency of monoallelic TSHR mutation (1:172 in Japan (10)) is much higher than NBS positivity (approximately 1:3000 in Japan), it is reasonably assumed that the proportion of monoallelic TSHR mutation carriers among NBS-positive individuals would be higher if monoallelic TSHR mutations cause TSH elevation with high penetrance. However, in fact, the proportion of monoallelic TSHR mutation carriers in NBSpositive individuals is only $3-12 \%(9,10)$. This indicates that only a minor fraction of monoallelic TSHR mutation carriers would have NBS positivity. Thus, we hypothesized that monoallelic TSHR mutations would not absolutely cause TSH elevation, but act as one of the multiple risk factors, modifying the probability of NBS positivity.

Dual oxidase 2 (DUOX2) is a transmembrane protein expressed on the apical membrane of thyroid follicular cells. DUOX2 produces hydrogen peroxide $\left(\mathrm{H}_{2} \mathrm{O}_{2}\right)$, which is required for iodination of thyroglobulin. DUOX2 gene mutations, which follow autosomal recessive inheritance, are the most common genetic cause of $\mathrm{CH}$ in Japan (14). Recently, an NBS-positive case with monoallelic TSHR mutation and monoallelic DUOX2 mutation in the same individual has been reported (15), but the biological effect of coexistence of these two monoallelic mutations has not been quantitatively clarified.

In this study, to demonstrate the significance of monoallelic TSHR mutations in $\mathrm{CH}$, we applied statistical approaches (e.g., odds ratio (OR) and Bayes' theorem (16)) that are typically used to analyze multifactorial diseases in which multiple factors contribute to disease risk with smaller effects $(17,18,19)$.

\section{Subjects and methods}

\section{Study subjects}

$\mathrm{CH}$ is a heterogeneous disease with various degrees of severity. Hence, definition of $\mathrm{CH}$ can be arbitrary and varies between the studies. In the present study, for simplicity, we defined CH by NBS positivity with following two criteria: (i) having a positive result in NBS and (ii) having serum TSH level equal or more than $5 \mathrm{mU} / \mathrm{L}$ at least once. Thus, the study subjects include permanent $\mathrm{CH}$ and transient $\mathrm{CH}$. We exclude patients with chromosomal abnormalities, central hypothyroidism or offspring of mothers with Graves' disease.

This study was approved by the Ethics Committee of Keio University School of Medicine. We obtained written informed consent for participating in the study from the subjects and/or their parents. Peripheral blood samples derived from 395 primary $\mathrm{CH}$ patients (belonging to 368 families) were sent to us for molecular diagnosis of genetic $\mathrm{CH}$ from 33 institutions in Japan. Out of the 368 families, 19 families had two $\mathrm{CH}$ patients and four families had three $\mathrm{CH}$ patients. This cohort includes 102 patients that have been previously reported $(10,14,20)$.

\section{Mutation detection}

For all subjects, all 10 coding exons and flanking introns of TSHR were analyzed by PCR-based direct sequencing as previously described (10). As for monoallelic TSHR mutation carriers, we further sequenced all 33 coding exons and flanking introns of DUOX2 as previously described (14). The detected variants were referred for the Human Genetic Variation Database (http://www. genome.med.kyoto-u.ac.jp/SnpDB/; Japanese genetic variant database), the 1000 Genomes Project database (http://www.1000genomes.org/) and the ESP6500 exome database (http://evs.gs.washington.edu/EVS/). We regarded a variant as benign polymorphism, if the allele frequency of the variant exceeds 0.01 in one or more of the databases.

As for DUOX2 p.H678R, which is a functional SNP with allele frequency 0.035 (14), we conducted statistical analyses under two conditions (exclude or include the variant as a mutation). In this article, we show the data excluding the DUOX2 p.H678R. The data including the variant, providing similar results, are shown in the Supplementary data published as Supplementary data given at the end of this article. 


\section{Functional assessment of TSHR variants and DUOX2 variants}

Detailed experimental procedures are available in the Supplementary data. To verify the pathogenicity of previously uncharacterized amino acid-altering variants with allele frequency $<0.01$, we conducted expression experiments using human embryonic kidney 293 cells.

The expression vectors for human TSHR, DUOX2 and dual oxidase maturation factor 2 (DUOXA2) have been reported previously $(10,14)$. The DUOXA2 is required for the maturation and transport of DUOX2 from the endoplasmic reticulum to the plasma membrane. The expression vectors for R519H-TSHR, L669H-TSHR, A705Dfs*24-TSHR, E327*-DUOX2, K530*-DUOX2 and V779M-DUOX2 were created by site-direct mutagenesis (QuickChange II XL Site-Direct Mutagenesis Kit; Agilent). All final constructs were verified by direct DNA sequencing.

For assessment of TSHR variants, TSH-dependent activation of Gs-coupled signal transduction was studied with a luciferase reporter responding to intracellular cAMP level (pGL4.29; Promega). For assessment of DUOX2 variants, $\mathrm{H}_{2} \mathrm{O}_{2}$-producing capacities were studied in the presence of cotransfected DUOXA2 with the use of the Amplex Red Reagent (Life Technologies).

The activity of each variant was expressed as the percentage (mean \pm s.e.m.) of wildtype activity. Data are representative of three independent experiments (each performed in triplicate) with similar results. Welch's $t$-test was used for comparisons of the activities. $P<0.05$ was considered statistically significant.

\section{The frequency of monoallelic TSHR mutation carriers and double heterozygotes (monoallelic TSHR mutation carriers that had an additional monoallelic DUOX2 mutation) in the Japanese general population}

We previously estimated the frequency of monoallelic TSHR mutation carriers in the Japanese general population as $0.58 \%(1: 172)$ by applying the Hardy-Weinberg equation to the frequency of biallelic TSHR mutations (10). The frequency of permanent $\mathrm{CH}$ or transient $\mathrm{CH}$ due to biallelic DUOX2 mutations has been reported to be 1:44 000 or 1:29 600 , respectively $(14,21)$. We calculated the frequency of double heterozygotes in the Japanese general population by multiplication of the frequencies of monoallelic TSHR mutation carriers and DUOX2 mutation carriers including permanent $\mathrm{CH}$ and transient $\mathrm{CH}$, providing the estimated frequency of $0.0087 \%$ (Supplementary data).

\section{Statistical analyses}

For our analysis, we assumed that the probability of NBS positivity was $1: 2975$, which is based on the observation of the frequency within the general population of Japan. These data were derived from Kanagawa prefecture, Japan, collected from 1979 to 2014 (838/2 493 200, our unpublished data). The frequency of patients with each mutation in the $\mathrm{CH}$ patients and its exact $95 \%$ confidence interval (CI) were estimated. One sample $Z$-test was used to compare the frequency in $\mathrm{CH}$ patient population $P$ with the theoretical value $P_{0}$ in the general population $\left(\mathrm{H}_{0}: P=P_{0}\right)$. We calculated the OR for NBS positivity associated with the mutation status (monoallelic TSHR mutation; double heterozygotes) by two-by-two tables. We estimated the $95 \%$ CI for the OR using a normal approximation by the delta method. To estimate the posterior probability of NBS positivity in mutation carriers, we used Bayes' theorem $(16,17)$. We defined the parameters as follows:

$P(X)$ : probability of $X$

$P(Y)$ : probability of $Y$

$P(X \mid Y)$ : conditional probability of $X$ given $Y$

By Bayes' theorem,

$$
P(X \mid Y)=\frac{P(Y \mid X) P(X)}{P(Y)}
$$

We estimated the posterior probability of NBS positivity in monoallelic TSHR mutation carriers ( $X$ : NBS positivity, $Y$ : carrying monoallelic TSHR mutation) and the posterior probability of NBS positivity in double heterozygotes ( $X$ : NBS positivity, $Y$ : carrying double heterozygous mutations).

The difference in serum TSH levels and in serum-free thyroxine (FT4) level were examined using the MannWhitney $U$ test, only in those groups with $N>5$.

\section{Results}

\section{Characteristics of the subjects}

In this study, $395 \mathrm{CH}$ patients (192 males, 203 females) were enrolled. The characteristics of the subjects are summarized in Table 1 . The median age at the last observation was 12.9 years. The 58 patients had a family history of $\mathrm{CH}$. Thyroid dysgenesis (i.e. thyroid ectopia, aplasia and hypoplasia) was observed in 121 patients. Thirty patients had goiter and 153 patients had in situ normal-sized thyroid gland. Thyroid morphology was not 
Table 1 Characteristics of the study subjects.

\begin{tabular}{l} 
Variable \\
\hline$n$ (males/females) \\
Age at last observation, median (IQR) (year) \\
Family history of CH $(n)$ \\
Blood-spot TSH at screening, median (IQR) (mU/L) \\
Serum TSH at reevaluation, median (IQR) (mU/L) \\
Serum FT4 at initial evaluation, median (IQR) (ng/dL) \\
Serum FT4 at reevaluation, median (IQR) (ng/dL) \\
Thyroid morphology $(n)$ \\
$\quad$ Ectopia \\
$\quad$ Aplasia \\
$\quad$ Hypoplasia \\
$\quad$ Goiter \\
$\quad$ Normal \\
$\quad$ Not evaluated \\
Thyroid size ${ }^{\text {d }}$ (s.D.), median (IQR)
\end{tabular}

\begin{tabular}{c}
\hline \\
\hline $395(192 / 203)$ \\
$12.9(8.8-18.0)$ \\
58 \\
$38.9(21.0-83.8)$ \\
$8.6(4.3-54.9)$ \\
$1.1(0.7-1.4)$ \\
$1.2(0.6-1.3)$ \\
52 \\
30 \\
39 \\
30 \\
153 \\
91 \\
$-0.7(-1.6$ to 1.0$)$
\end{tabular}

${ }^{a}$ Thyroid morphology was evaluated by ultrasonography or scintigraphy; ${ }^{b}$ definition of hypoplasia is smaller than -2 s.D. of normal thyroid size; 'definition of goiter is larger than 2 S.D. of it; d'thyroid sizes were measured by ultrasonography in 139 patients (22). IQR, interquartile range.

evaluated in 91 patients. The thyroid sizes were measured by ultrasonography in 139 patients (22). Median bloodspot TSH level on NBS was $38.9 \mathrm{mU} / \mathrm{L}$. The median serum TSH level at reevaluation with stopping therapy at age 1 or more year was $8.6 \mathrm{mU} / \mathrm{L}$. Two hundred seventy-eight patients had permanent $\mathrm{CH}, 68$ had transient $\mathrm{CH}$ and 49 patients lacked data about type of $\mathrm{CH}$.

\section{Detection and functional assessment of TSHR mutations and DUOX2 mutations}

Using PCR-based direct sequencing for 395 subjects, we found nine biallelic TSHR mutation carriers (data not shown) and 26 monoallelic TSHR mutation carriers (Supplementary Fig. 1 and Table 1). In the 26 monoallelic mutation carriers, we detected five previously reported mutations (p.G132R (10), p.A204V (10), p.R450H (10), p.R519H (23) and p.A553T (5)) and two novel sequence variants (p.L669H and p.A705Dfs*24). In the three TSHR variants, TSH-dependent activation of the cAMP pathway was markedly lower than that in wildtype (Fig. 1A).

For the 26 patients with a monoallelic TSHR mutation, we further sequenced DUOX2. Surprisingly, we found that four out of 26 had an additional monoallelic DUOX2 mutation (double heterozygotes). In the four double heterozygotes, we detected three previously uncharacterized amino acid-altering variants (p.E327* (24), p.K530* $(25,26)$ and p.V779M (27)). The DUOX2 variant (V779M) was described as a splice variant in 1000 Genomes Project database (rs145061993, allele frequency of Japanese 0.007).
E327*-DUOX2 and K530*-DUOX2 had negligible $\mathrm{H}_{2} \mathrm{O}_{2}$-producing capacities. The V779M DUOX2 showed partial but statistically significant reduction in $\mathrm{H}_{2} \mathrm{O}_{2}$ producing activity $(76 \pm 14 \%, P=0.02$ by Welch's $t$-test) (Fig. 1B). Because of splice site variant, the V779M is estimated to produce abnormal protein or not be able to produce the protein.

\section{Clinical phenotypes of the mutation carriers}

The 22 monoallelic TSHR mutation carriers without monoallelic DUOX2 mutations had relatively uniform clinical phenotypes (Supplementary Table 1). There were two sets of siblings (from two separate families) with
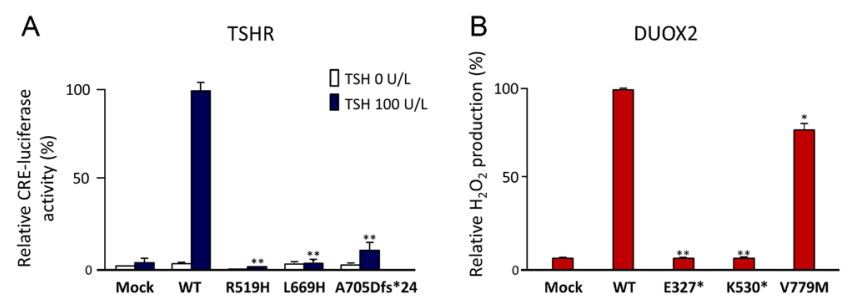

\section{Figure 1}

Functional properties of three TSHR mutations and three DUOX2 mutations. (A) TSH-dependent CAMP response element (CRE) activation was studied. (B) $\mathrm{H}_{2} \mathrm{O}_{2}$-producing capacities of DUOX2 proteins were measured. Data are representative of three independent experiments with similar results. Values are mean \pm S.E.M. ${ }^{*} P<0.05$ and $* * P<0.01$ by Welch's $t$-test vs wildtype. A full colour version of this figure is available at https://doi.org/10.1530/EJE-16-1049. 
$\mathrm{CH}$. They had marginally small or normal-sized thyroid gland in situ, and their serum TSH levels at reevaluation were normal to slightly elevated (median: $6.3 \mathrm{mU} / \mathrm{L}$ (interquartile range: 4.4-9.3)). Their serum TSH levels initially and at reevaluation were significantly lower than those in the remaining patient group (Fig. 2A and B), while their FT4 levels initially and at reevaluation were higher than those in the remaining patient group (Fig. 2C and $\mathrm{D})$. The reference values depicted in the figure are from Nelson et al (28). 16 out of 22 were treated with levothyroxine, and four of them withdrew from treatment after reevaluation.

Patient 1 (a 5-year-old girl; TSHR p.R450H, DUOX2 p.E327*) was born after 38 weeks of gestation with birth weight $3108 \mathrm{~g}(+1.2$ s.D.). At initial evaluation, her serum TSH level was slightly high $(14.1 \mathrm{mU} / \mathrm{L})$ with a normal-serum FT4 level. She had mild intermittent hyperthyrotropinemia (ranging between 3.5 and $8.9 \mathrm{mU} / \mathrm{L}$ ), and had not been treated. At age 5 years, ultrasonography revealed marginally small thyroid gland in situ (-1.7s.D.). She presented normal growth and development.

Patients 2 and 3 (9-year-old boys; TSHR p.R450H, DUOX2 p.K530*) were monozygotic twins born after 33 weeks of gestation. Patient 2 was an appropriate-forgestational-age infant $(1850 \mathrm{~g},-0.3$ s.D. $)$, while Patient 3 was a small-for-gestational-age infant $(974 \mathrm{~g},-3.5$ s.D.). Blood-spot TSH levels of Patient 2 in NBS at age 30 days were slightly elevated $(14.0 \mathrm{mU} / \mathrm{L})$. Patient 2 had a normal-serum TSH level $(4.9 \mathrm{mU} / \mathrm{L})$ on first evaluation at age 40 days. He was not treated with levothyroxine due
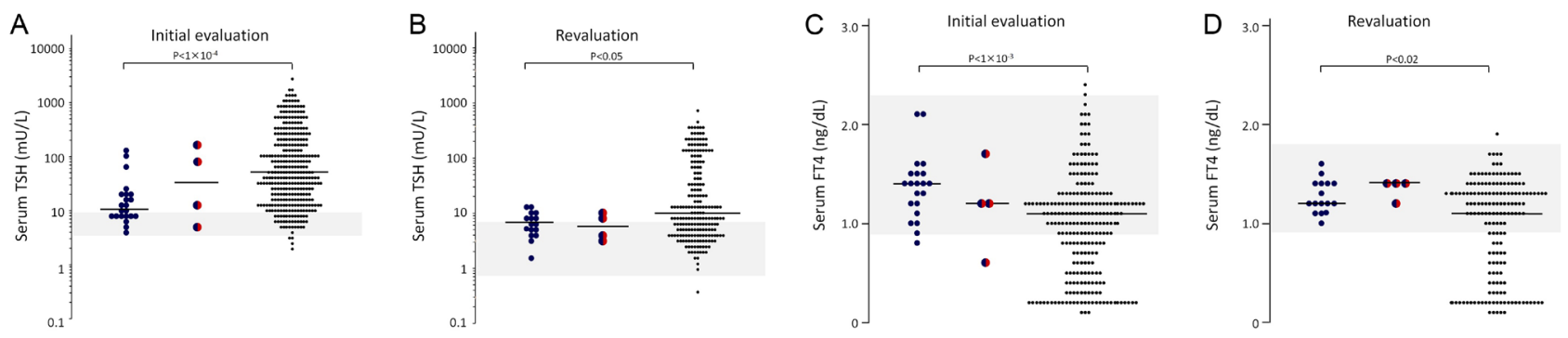

- Monoallelic TSHR mutation carriers

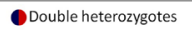

Remaining patients — Median to a persistent slight elevation of TSH (ranging between 1.4 and $7.2 \mathrm{mU} / \mathrm{L}$ ). He had marginally small thyroid on ultrasonography ( -1.9 s.D.) at age 1 year. Patient 3 was a donor of twin-to-twin transfusion syndrome and had signs of fetal distress. Thus, the twins were delivered by an emergency cesarean section at 33 weeks of gestation. Patient 3 had a high-serum TSH level $(85.3 \mathrm{mU} / \mathrm{L})$ on initial evaluation at age 37 days. He was treated with levothyroxine. At age 3 years, when we reevaluated his thyroid function with stopping therapy, he had a normal-serum TSH level $(3.3 \mathrm{mU} / \mathrm{L})$ and had a normal thyroid function thereafter. The thyroid size was normal (-0.4s.D.) by ultrasonography. Patients 2 and 3 were growing normally and were developing satisfactorily. A family analysis showed that their mother was a double heterozygote (Fig. 3 family 2 I-2). She did not have a history of thyroid disease. Her thyroid function was normal.

Patient 4 (an 11-year-old girl; TSHR p.R450H, DUOX2 p.V779M) presented with unidentified intrauterine growth retardation and was born as a small-forgestational-age infant $(534 \mathrm{~g},-3.4$ s.D. $)$ at 27 weeks of gestation. Her blood-spot TSH level at age 10 days was slightly elevated $(13.6 \mathrm{mU} / \mathrm{L}$; age-specific ref.: $<15)$ and serum FT4 was low $(0.8 \mathrm{ng} / \mathrm{dL}$; age-specific ref.: 0.9-2.3). Because she was extremely premature, this TSH level seemed to be hypothyroxinemia of prematurity. Her blood-spot TSH level, when she had rescreening at age 55 days, was elevated $(64.5 \mathrm{mU} / \mathrm{L})$. Her serum TSH level at age 60 days was also elevated $(164.5 \mathrm{mU} / \mathrm{L}$, regarded as initial evaluation at 36 weeks post-conceptual age) with a

Figure 2

Distribution of serum TSH and serum FT4 level. (A) The serum TSH levels at initial evaluation. (B) The serum TSH levels at reevaluation at age 1 or more year under levothyroxine withdrawal. (C) The serum FT4 levels at initial evaluation. (D) The serum FT4 levels at reevaluation. Serum TSH levels of monoallelic TSHR mutation carriers (blue) and double heterozygotes (blue and red) clustered around $10 \mathrm{mU} / \mathrm{L}$ at reevaluation, while those of remaining patients (black) had broader distribution. We used the Mann-Whitney $U$ test to compare of serum TSH and FT4 levels between monoallelic TSHR mutation carriers and remaining patients. Double heterozygotes were excluded from the test because $n<5$. Reference values (gray shade) are from Nelson and coworkers (28). 


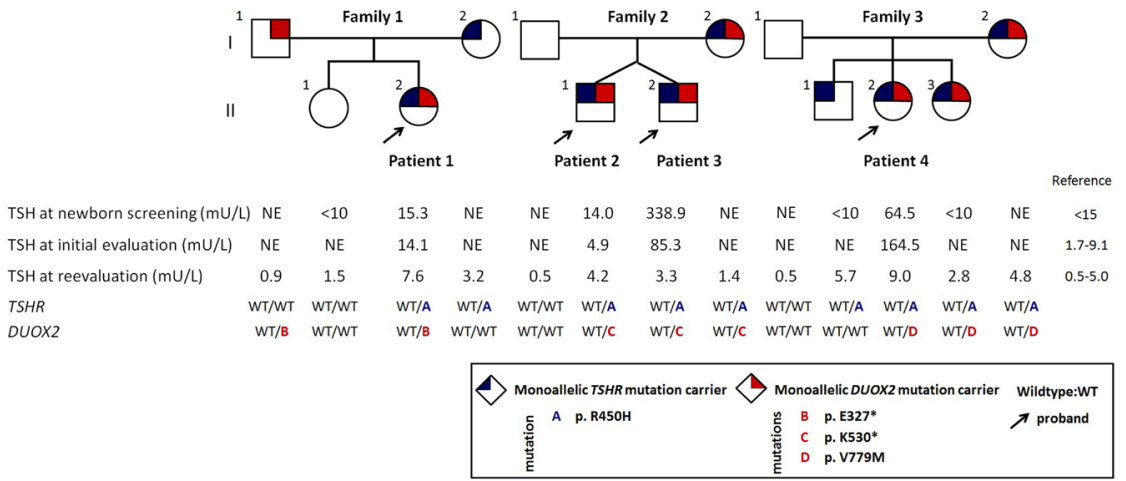

Figure 3

Pedigrees of the four double heterozygotes. Square symbols indicate males; circles indicate females. The genotype of each subject is indicated within the symbol, as identified in the legend below the pedigree. Results of TSH levels (at newborn screening and at reevaluation) are aligned with each symbol and were obtained on no levothyroxine replacement. NE, not evaluated. normal FT4 level (1.2 ng/dL). She received levothyroxine replacement therapy immediately after the diagnosis of $\mathrm{CH}$. At age 6 years, reevaluation of thyroid function with stopping therapy showed a slightly high-serum TSH level $(9.0 \mathrm{mU} / \mathrm{L})$ with a normal-serum FT4 level. She did not need levothyroxine treatment thereafter, but had mild intermittent hyperthyrotropinemia (ranging between 4.8 and $7.0 \mathrm{mU} / \mathrm{L})$. Her thyroid was small (-2.7s.D.) on ultrasonography at age 6 years. She was growing normally and was developing satisfactorily.

Family studies of the double heterozygotes revealed three additional double heterozygotes (Fig. 3 family 2 I-2, family 3 I-2, II-3). They did not have a history of thyroid diseases and had normal thyroid function.

\section{Statistical analyses of monoallelic TSHR mutations and double heterozygotes}

The frequency of monoallelic TSHR mutation carriers in the $\mathrm{CH}$ patient cohort was $6.6 \%$ (26 out of $395 \mathrm{CH}$ patients) (95\% CI, 4.3-9.5\%), which was significantly higher than that in the Japanese general population $(P<0.001, Z$-test for $\mathrm{H}_{0}$ : true frequency $=0.58 \%$ ) (Supplementary Fig. 2A). The OR for NBS positivity associated with carrying a monoallelic TSHR mutation was 12.0 (95\% CI, 8.1-18.0). As for double heterozygotes, the frequency in $\mathrm{CH}$ patients was $1.0 \%$ (4 out of $395 \mathrm{CH}$ patients) (95\% CI, 0.28$2.6 \%)$, and it was significantly higher than that in the Japanese general population $\left(P<0.05, Z\right.$-test for $\mathrm{H}_{0}$ : true frequency $=0.0087 \%$ ) (Supplementary Fig. 2A). The OR for NBS positivity associated with double heterozygotes was 117.9 (95\% CI, 13.1-1057.2). Here, the 95\% CI for the OR was shown as reference, although the prevalence in non-CH population, namely in the Japanese general population, was predicted from an external research (10, $14,21)$. Next, we analyzed the frequency of monoallelic TSHR mutations and double heterozygotes in the context of its distribution in families. We did this analysis because some of our research subjects are siblings (19 families had $2 \mathrm{CH}$ patients and four families had $3 \mathrm{CH}$ patients out of a total of 368 families). The frequency of monoallelic TSHR mutation was $6.3 \%$ (23 out of 368 families) and of double heterozygotes was $0.8 \%$ (3 out of 368 families). From this analysis, we estimated the OR associated with monoallelic TSHR mutation or double heterozygotes as 11.4 (95\% CI, 7.5-17.4) or 94.7 (95\% CI, 9.8-912.7), respectively. These results of the OR estimated treating family as a unit were comparable to those in entire population treating each patient to be independent.

As for all $\mathrm{CH}$ patient cohort (395 $\mathrm{CH}$ patients), we estimated a posterior conditional probability of NBS positivity given the monoallelic TSHR mutation and double heterozygotes by Bayes' theorem. A posterior conditional probability of NBS positivity given the monoallelic TSHR mutation (26 mutation-positive patients out of $395 \mathrm{CH}$ patients) and the probability of NBS positivity given the double heterozygotes (4 mutation-positive patients out of $395 \mathrm{CH}$ patients) was $0.38 \%$ and $3.8 \%$, respectively (Supplementary data for details of the calculation) (Supplementary Fig. 2B).

\section{Discussion}

In the present study, we used a statistical approach based on the frequencies of mutation carriers in a $\mathrm{CH}$ patient cohort and the Japanese general population to define the pathological role of monoallelic TSHR mutations. We demonstrated that monoallelic TSHR mutations are actually associated with NBS positivity, increasing the probability by 12 times. However, due to the rarity of $\mathrm{CH}$ (about 1 in 3000), the estimated probability of NBS positivity in monoallelic TSHR mutation carriers was fairly low $(0.38 \%)$. These data would be useful for conducting more appropriate genetic counseling. 
Biallelic-inactivating TSHR mutation carriers invariably develop $\mathrm{CH}$, while only a minor subset of monoallelic mutation carriers does. It is reasonable to speculate that monoallelic mutations in TSHR, which act as a sensor for TSH, shifted the distribution of serum TSH levels toward higher values (Supplementary Fig. 3). Consistent with the shift-distribution model, few monoallelic TSHR mutation carriers found in the present study had overt hypothyroidism at reevaluation (Fig. 2B). This 'distribution-shift' model also agrees well with the low estimated probability of $\mathrm{CH}$ affection in monoallelic TSHR mutation carriers $(0.38 \%)$, because the TSH levels of most monoallelic mutation carriers are expected to remain within normal range in the model (Supplementary Fig. 3).

The most significant observation of this study is unexpectedly high frequency of additional monoallelic DUOX2 mutations among monoallelic TSHR mutation carriers. OR for NBS positivity associated with double heterozygotes was 117.9 , which was 10 times higher than the one associated with a monoallelic TSHR mutation alone. These data can be interpreted as synergy between the two gene mutations. Since DUOX2 is the same thyroid hormone-producing pathway with TSHR, we speculated that NBS positivity may have increased synergistically. In this study, serum TSH levels of the double heterozygotes were variable in their neonatal period, implying the presence of additional factor(s) affecting the thyroid hormone-producing capacity after birth. For example, Patients 2 and 3 were monozygotic twins (namely identical genetic background), but the birth weight was substantially discordant. The serum TSH levels were also discordant between the two: Patient 3 with low birth weight had a higher serum TSH level than Patient 2. Similarly, Patient 4 was born as a premature infant and had $\mathrm{CH}$, while her sister, who was also a double heterozygote, was born at term and did not have $\mathrm{CH}$. These observations indicate that the onset and/or the severity of $\mathrm{CH}$ in double heterozygotes could be further influenced by prematurity at birth (29).

In our 'distribution-shift' model (Supplementary Fig. 3), monoallelic TSHR mutation carriers that had a positive result in NBS are at the right end of the distribution and would likely have additional factor(s) negatively affecting the thyroid hormone-producing capacity. We showed statistical and descriptive evidence that monoallelic DUOX2 mutations and prematurity at birth act as such factors. Another prediction from the 'distributionshift' model is that the disease would be mild in $\mathrm{CH}$ $(\mathrm{TSH}<10 \mathrm{mU} / \mathrm{L})$ due to accumulation of the risk factors (e.g., monoallelic mutations in the autosomal recessive loci and prematurity at birth). Consistent with the prediction, clinical phenotypes of the 26 monoallelic TSHR mutation carriers, including four double heterozygotes, were mild. These results indicate that the TSH elevation is more sensitively influenced by the other factor of monoallelic mutations in the neonatal period than in childhood.

In conclusion, we defined the pathologic role of monoallelic TSHR mutations by applying a statistical approach to a large-scale patient cohort. Monoallelic TSHR mutations are strongly associated with NBS positivity, but most monoallelic mutation carriers remain screening negative due to the rarity of $\mathrm{CH}$. We also showed that the probability of NBS positivity associated with monoallelic TSHR mutations is further modified by monoallelic DUOX2 mutations, and possibly by prematurity at birth. We presume that some portion of the etiology of mild $\mathrm{CH}$ is explained by the accumulation of the risk factors with a mild biological effect. A statistical framework would be further required for better delineation of the mechanisms underlying mild $\mathrm{CH}$.

\section{Supplementary data}

This is linked to the online version of the paper at https://doi.org/10.1530/ EJE-16-1049.

Declaration of interest

The authors declare that there is no conflict of interest that could be perceived as prejudicing the impartiality of the clinical study.

\section{Funding}

This work was supported by JSPS KAKENHI (Grant Numbers JP24791088, JP16K19560 and JP15K09630) and a grant from the Ministry of Health, Labour and Welfare, Japan (Jitsuyoka (Nanbyo)-Ippan-014). The grant from Kawano Masanori Memorial Public Interest Incorporated Foundation for Promotion of Pediatrics also supported this work.

Author contribution statement

$\mathrm{K}$ Abe, $\mathrm{S}$ Narumi and T Hasegawa conceived and designed the experiments, $\mathrm{K}$ Abe and $\mathrm{S}$ Narumi performed the experiments, $\mathrm{K}$ Abe analyzed the experimental data, $\mathrm{K}$ Abe and $\mathrm{T}$ Abe analyzed the statistical data, $\mathrm{A}$ Suwanai, M Adachi, K Muroya, Y Asakura and K Nagasaki contributed for materials and clinical data and K Abe, S Narumi and T Hasegawa wrote the paper.

\section{Acknowledgements}

The authors are grateful to Prof. Takao Takahashi for the fruitful discussion.

\section{References}

1 Toublanc JE. Comparison of epidemiological data on congenital hypothyroidism in Europe with those of other parts in the world. Hormone Research 199238 230-235. (doi:10.1159/000182549)

2 Deladoey J, Ruel J, Giguere Y \& Van Vliet G. Is the incidence of congenital hypothyroidism really increasing? A 20-year retrospective population-based study in Quebec. Journal of Clinical Endocrinology and Metabolism 201196 2422-2429. (doi:10.1210/jc.2011-1073) 
3 Sunthornthepvarakui T, Gottschalk ME, Hayashi Y \& Refetoff S. Brief report: resistance to thyrotropin caused by mutations in the thyrotropin-receptor gene. New England Journal of Medicine 1995332 155-160. (doi:10.1056/NEJM199501193320305)

4 Persani L, Calebiro D, Cordella D, Weber G, Gelmini G, Libri D, de Filippis T \& Bonomi M. Genetics and phenomics of hypothyroidism due to TSH resistance. Molecular and Cellular Endocrinology 2010322 72-82. (doi:10.1016/j.mce.2010.01.008)

5 Abramowicz MJ, Duprez L, Parma J, Vassart G \& Heinrichs C. Familial congenital hypothyroidism due to inactivating mutation of the thyrotropin receptor causing profound hypoplasia of the thyroid gland. Journal of Clinical Investigation 199799 3018-3024. (doi:10.1172/JCI119497)

6 Grasberger H, Van Sande J, Hag-Dahood Mahameed A, TenenbaumRakover Y \& Refetoff S. A familial thyrotropin (TSH) receptor mutation provides in vivo evidence that the inositol phosphates/ $\mathrm{Ca} 2+$ cascade mediates TSH action on thyroid hormone synthesis. Journal of Clinical Endocrinology and Metabolism 200792 2816-2820. (doi:10.1210/jc.2007-0366)

7 Nicoletti A, Bal M, De Marco G, Baldazzi L, Agretti P, Menabo S, Ballarini E, Cicognani A, Tonacchera M \& Cassio A. Thyrotropinstimulating hormone receptor gene analysis in pediatric patients with non-autoimmune subclinical hypothyroidism. Journal of Clinical Endocrinology and Metabolism 200994 4187-4194. (doi:10.1210/ jc.2009-0618)

8 Alberti L, Proverbio MC, Costagliola S, Romoli R, Boldrighini B, Vigone MC, Weber G, Chiumello G, Beck-Peccoz P \& Persani L. Germline mutations of TSH receptor gene as cause of non-autoimmune subclinical hypothyroidism. Journal of Clinical Endocrinology and Metabolism 200287 2549-2555. (doi:10.1210/jcem.87.6.8536)

9 Calebiro D, Gelmini G, Cordella D, Bonomi M, Winkler F, Biebermann H, de Marco A, Marelli F, Libri DV, Antonica F et al. Frequent TSH receptor genetic alterations with variable signaling impairment in a large series of children with non-autoimmune isolated hyperthyrotropinemia. Journal of Clinical Endocrinology and Metabolism 201297 E156-E160. (doi:10.1210/jc.2011-1938)

10 Narumi S, Muroya K, Abe Y, Yasui M, Asakura Y, Adachi M $\&$ Hasegawa T. TSHR mutations as a cause of congenital hypothyroidism in Japan: a population-based genetic epidemiology study. Journal of Clinical Endocrinology and Metabolism 200994 1317-1323. (doi:10.1210/jc.2008-1767)

11 Rapa A, Monzani A, Moia S, Vivenza D, Bellone S, Petri A, Teofoli F, Cassio A, Cesaretti G, Corrias A et al. Subclinical hypothyroidism in children and adolescents: a wide range of clinical, biochemical, and genetic factors involved. Journal of Clinical Endocrinology and Metabolism 200994 2414-2420. (doi:10.1210/jc.2009-0375)

12 Calebiro D, de Filippis T, Lucchi S, Covino C, Panigone S, BeckPeccoz P, Dunlap D \& Persani L. Intracellular entrapment of wild-type TSH receptor by oligomerization with mutants linked to dominant TSH resistance. Human Molecular Genetics 200514 2991-3002. (doi:10.1093/hmg/ddi329)

13 Grasberger H, Mimouni-Bloch A, Vantyghem MC, van Vliet G, Abramowicz M, Metzger DL, Abdullatif H, Rydlewski C, Macchia PE, Scherberg NH et al. Autosomal dominant resistance to thyrotropin as a distinct entity in five multigenerational kindreds: clinical characterization and exclusion of candidate loci. Journal of Clinical Endocrinology and Metabolism 200590 4025-4034. (doi:10.1210/ jc.2005-0572)

14 Narumi S, Muroya K, Asakura Y, Aachi M \& Hasegawa T. Molecular basis of thyroid dyshormonogenesis: genetic screening in population-based Japanese patients. Journal of Clinical Endocrinology and Metabolism 201196 E1838-E1842. (doi:10.1210/jc.2011-1573)
15 Satoh M, Aso K, Ogikubo S, Yoshizawa-Ogasawara A \& Saji T. Hypothyroidism caused by the combination of two heterozygous mutations: one in the TSH receptor gene the other in the DUOX2 gene. Journal of Pediatric Endocrinology and Metabolism 201528 657-661. (doi:10.1515/jpem-2014-0078)

16 Carlin BP \& Louis TA. Bayesian Methods for Data Analysis. Boca Raton, Florida: Chapman and Hall/CRC Texts in Statistical Science, 2008. (doi:10.1007/s11336-010-9148-6)

17 Berry DA, Parmigiani G, Sanchez J, Schildkraut J \& Winer E. Probability of carrying a mutation of Breast-Ovarian Cancer Gene BRCA1 based on family history. Journal of the National Cancer Institue 199789 227-237. (doi:10.1093/jnci/89.3.227)

18 Dunson DB. Commentary: practical advantages of Bayesian analysis of epidemiologic data. American Journal of Epidemiology 2001153 1222-1226. (doi:10.1093/aje/153.12.1222)

19 Petersen GM, Parmigiani G \& Thomas D. Missense mutations in disease genes: a Bayesian approach to evaluate causality. American Journal of Human Genetics 199862 1516-1524. (doi:10.1086/301871)

20 Narumi S, Muroya K, Asakura Y, Adachi M \& Hasegawa T. Transcription factor mutations and congenital hypothyroidism: systematic genetic screening of a population-based cohort of Japanese patients. Journal of Clinical Endocrinology and Metabolism 201095 1981-1985. (doi:10.1210/jc.2009-2373)

21 Nagasaki K, Narumi S, Abe K, Asami T, Sato H, Ogawa Y, Kikuchi T, Hasegawa T \& Saitoh A. A study of the etiology of transient congenital hypothyroidism in Niigata Prefecture, Japan. International Journal of Pediatric Endocrinology 20132013 (Supplement 1) O55. (doi:10.1186/1687-9856-2013-S1-O55)

22 Yasumoto M, Inoue H, Ohashi I, Shibuya H \& Onishi T. Simple new technique for sonographic measurement of the thyroid in neonates and small children. Journal of Clinical Ultrasound 200432 82-85. (doi:10.1002/jcu.10234)

23 Park KJ, Park S, Lee E, Park JH, Park JH, Park HD, Lee SY \& Kim JW. A population-based genomic study of inherited metabolic diseases detected through newborn screening. Annals of Laboratory Medicine 201636 561-572. (doi:10.3343/alm.2016.36.6.561)

24 Kasahara T, Narumi S, Okasora K, Takaya R, Tamai H \& Hasegawa T. Delayed onset congenital hypothyroidism in a patient with DUOX2 mutations and maternal iodine excess. American Journal of Medical Genetics Part A 2013 161A 214-217. (doi:10.1002/ajmg.a.35693)

$25 \mathrm{Fu} \mathrm{C}$, Luo S, Zhang S, Wang J, Zheng H, Yang Q, Xie B, Hu X, Fan $\mathrm{X}$, Luo $\mathrm{J}$ et al. Next-generation sequencing analysis of DUOX2 in 192 Chinese subclinical congenital hypothyroidism (SCH) and CH patients. Clinica Chimica Acta 2016458 30-34. (doi:10.1016/j. cca.2016.04.019)

26 Tan M, Huang Y, Jiang X, Li P, Tang C, Jia X, Chen Q, Chen W, Sheng H, Feng Y et al. The prevalence, clinical, and molecular characteristics of congenital hypothyroidism caused by DUOX2 mutations: a population-based cohort study in Guangzhou. Hormone and Metabolic Research 201648 581-588. (doi:10.1055/s-0042-112224)

27 Park KJ, Park HK, Kim YJ, Lee KR, Park JH, Park JH, Park HD, Lee SY \& Kim JW. DUOX2 mutations are frequently associated with congenital hypothyroidism in the Korean population. Annals of Laboratory Medicine 201636 145-153. (doi:10.3343/alm.2016.36.2.145)

28 Nelson JC, Clark SJ, Borut DL, Tomei RT \& Carlton EI. Age-related changes in serum free thyroxine during childhood and adolescence. Journal of Pediatrics 1993123 899-905. (doi:10.1016/S00223476(05)80385-3)

29 Radetti G, Renzullo L, Gottardi E, D’Addato G \& Messner H. Altered thyroid and adrenal function in children born at term and preterm, small for gestational age. Journal of Clinical Endocrinology and Metabolism 200489 6320-6324. (doi:10.1210/jc.2003-032185)

Received 20 December 2016

Revised version received 30 September 2017

Accepted 1 November 2017 